\title{
Effect of particle degradation on electrostatic sensor measurements and flow characteristics in dilute pneumatic conveying
}

\author{
Wei Chen ${ }^{1, *}$, Jianyong Zhang ${ }^{2}$, Timothy Donohue ${ }^{1}$, Kenneth Williams ${ }^{1}$, Ruixue Cheng ${ }^{2}$, Mark \\ Jones $^{1}$, Bin Zhou ${ }^{3}$ \\ ${ }^{1}$ Centre for Bulk Solids and Particulate Technologies, The University of Newcastle, Callaghan 2308, Australia \\ ${ }^{2}$ University of Teesside, Middlesbrough, Cleveland TS1, United Kingdom \\ ${ }^{3}$ Southeast University, Nanjing 210009, China \\ *Corresponding author: E-mail: W.Chen@newcastle.edu.au,W.Chentbs@gmail.com
}

\begin{abstract}
Vigorous particle collisions and mechanical processes occurring during high-velocity pneumatic conveying often lead to particle degradation. The resulting particle size reduction and particle number increase will impact on the flow characteristics, and subsequently affect the electrostatic type of flow measurements. This study investigates this phenomenon using both experimental and numerical methods. Particle degradation was induced experimentally, by recursively conveying the fillite material within a pneumatic pipeline. The associated particle size reduction was monitored. Three electrostatic sensors were embedded along the pipeline to monitor the flow. The results indicated a decreasing trend in the electrostatic sensor outputs with decreasing particle size, which suggested the attenuation of the flow velocity fluctuation. This trend was more apparent at higher conveying velocities, which suggested that more severe particle degradation occurred under these conditions. Coupled computational fluid dynamics and discrete element methods (CFDDEM) analysis was used to qualitatively validate these experimental results. The numerical results suggested that smaller particles exhibited lower flow velocity fluctuations, which was consistent with the observed experimental results. These findings provide important information for the accurate application of electrostatic measurement devices in pneumatic conveyors.
\end{abstract}

Keywords: Particle degradation, Flow velocity fluctuation, Electrostatic sensor, CFD-DEM modelling, Pneumatic conveying

\section{Nomenclature}

$\begin{array}{ll}C_{\mathrm{D}} & \text { drag coefficient } \\ d_{50} & \text { mean particle size }(\mu \mathrm{m}) \\ d_{\mathrm{p}} & \text { particle diameter }(\mathrm{m}) \\ \mathbf{F}_{\mathrm{b}} & \text { buoyance force }(\mathrm{N}) \\ \mathbf{F}_{\mathrm{c}} & \text { contact force }(\mathrm{N}) \\ \mathbf{F}_{\mathrm{d}} & \text { drag force }(\mathrm{N}) \\ \mathbf{F}_{\mathrm{f}} & \text { air-particle interphase momentum transfer force }(\mathrm{N}) \\ \mathbf{F}_{\mathrm{g}} & \text { gravitational force }(\mathrm{N})\end{array}$




$\begin{array}{ll}g & \text { gravitational acceleration }\left(\mathrm{m} / \mathrm{s}^{2}\right) \\ I & \text { particle inertia }\left(\mathrm{kg} \cdot \mathrm{m}^{2}\right) \\ \mathbf{M} & \text { contact torque }(\mathrm{N} \cdot \mathrm{m}) \\ m & \text { particle mass }(\mathrm{kg}) \\ P & \text { air pressure }(\mathrm{Pa}) \\ R e & \text { Reynolds number } \\ \mathbf{U}_{\mathrm{f}} & \text { fluid velocity }(\mathrm{m} / \mathrm{s}) \\ \mathbf{U}_{\mathrm{p}} & \text { particle translational velocity }(\mathrm{m} / \mathrm{s}) \\ \widehat{U}_{\mathrm{rms}} & \text { root mean square value of the signal } \\ \Delta \bar{U} & \text { signal variation } \\ \varepsilon & \text { void fraction } \\ \rho_{\mathrm{a}} & \text { air density }\left(\mathrm{kg} / \mathrm{m}^{3}\right) \\ \mu_{\mathrm{a}} & \text { air viscosity }(\mathrm{Pa} \cdot \mathrm{s}) \\ \boldsymbol{\omega} & \text { particle angular velocity }(\mathrm{m} / \mathrm{s})\end{array}$

\section{Introduction}

In pneumatic conveying, the flow rates of solid materials often need to be monitored or controlled. Accurate measurements of the flow velocity, concentration, and flow rate are critical. Electrostatic, electrical capacitance, and microwave measurements are three common non-invasive measurement techniques used for these purposes (Arko et al., 1999; Beck, Green, \& Thorn, 1987; Xu, Zhou, \& Wang, 2010; Yan, Byrne, \& Coulthard, 1995; Yan, 1996). The electrostatic method measures the flow rate by detecting induced charges carried by solid particles (Saleh \& Aghili, 2012; Zhang, Coulthard, Cheng, \& Keech, 2009; Zhang \& Coulthard, 2005). The primary sources of this electrification are frictional contact charging between particles or between particles and the conducting facility, charge transfer or sharing from one particle to another, and charge induction (Farmer, 1992; Zhou, Zhang, Xu, \& Wang, 2011).

In reality, particles can be positively and negatively charged during pneumatic transport. Fig. 1(a) schematically demonstrates the principle of this technique. Charge induction occurs at the inner surface of the earthed metal pipe wall and the insulated floating metal electrode. The conditioning circuit is used to detect charge induction on the electrode only. The output of the conditioning circuit can be used to indirectly indicate the solids flow rate (Gajewski \& Szaynok, 1981; Masuda, Komatsu, Mitsui, \& Iinoya, 1977). In industrial environments, electrostatic sensors are susceptible to low frequency noise. Hence, dynamic measurements are usually used, which measure the fluctuation of the charge or voltage induced in the electrode. The fluctuation of the signal (root mean square value is often used) has been used to indicate the solids flow velocity/flow rate. The signal level and its frequency band largely depend on the dynamic flow velocity fluctuation (Cole, Baum, \& Mobbs, 1969; Gajewski, 1997; King, 1973). This is demonstrated in Fig. 1(b), in which higher flow velocity fluctuations lead to an increase in the intensity and frequency of the signal. 


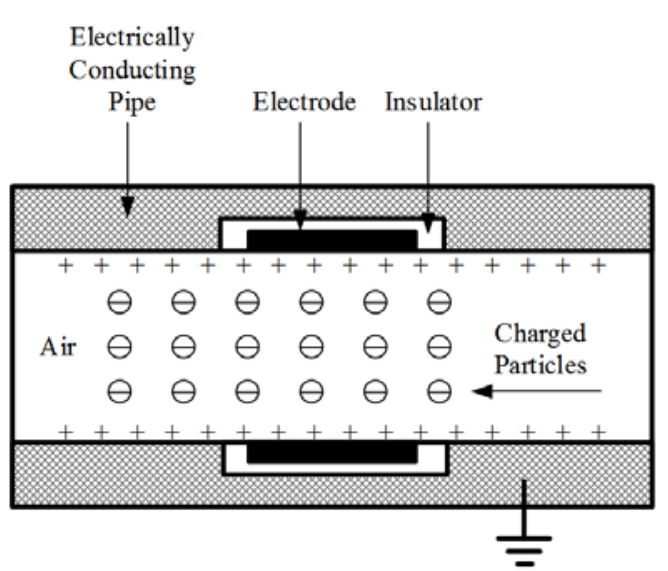

(a)

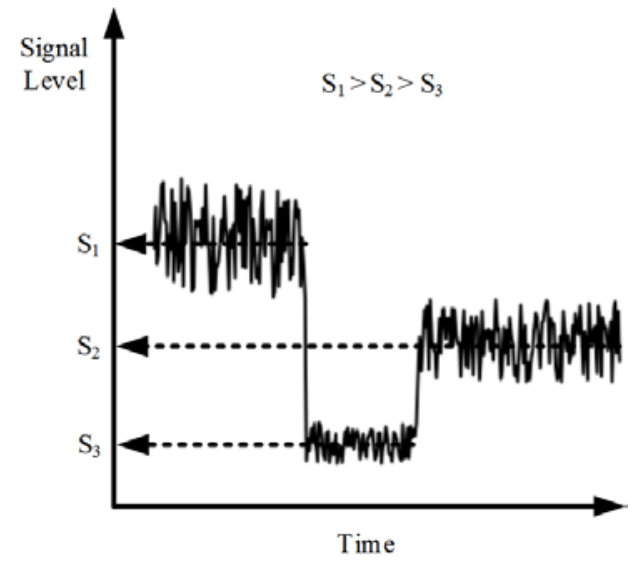

(b)

Fig. 1. Electrostatic flow sensor: (a) schematic of the measurement principle and (b) typical sensor output.

In dilute gas-solid two-phase flows, the high conveying velocity means that particle degradation/attrition often occurs from recursive particle-particle and particle-wall collisions. This results in: (1) a particle size reduction as the conveying process progresses. Therefore, the total number and total surface area of particles continuously increase for a given mass of material (Chapelle et al., 2004; Kalman, 2000; Mills, Jones, \& Agarwal, 2004; Salman, Attila, \& Mills, 1992); (2) a decrease in the flow velocity fluctuation. The increasing number of particles enhances the momentum dissipation, which leads to less chaotic velocity variations (Crowe, 2000; Hetsroni, 1989; Yarin \& Hetsroni, 1994).

The above two phenomena have opposite effects on the output of electrostatic dynamic sensors. Specifically, an increase in the particle surface area leads to a higher total charge carried by the particles, which will enhance the signal. Conversely, the decreasing flow velocity fluctuation will result in a weaker signal. The overall signal trend (rise or fall) depends on the balance between these two effects. This paper presents experimental results and qualitative simulations on the change in flow characteristics resulting from particle degradation. The experimental and simulated results are consistent with each other.

\section{Experimental scheme}

The experimental pneumatic conveying system used in this study is shown schematically in Fig. 2. The $40 \mathrm{~mm}$ (internal diameter) pipeline system consisted of one vertical and two horizontal sections. Three electrostatic meters with a same internal bore were installed along the pipe. A draft fan and an air drier were used to provide air to transport the solids fed from the screw feeder. Underneath the screw feeder, a weighing platform measured the mass of the solids, from which the solids mass flow rate was derived. After completing each conveying loop, the solids and air were separated in the cyclone. 


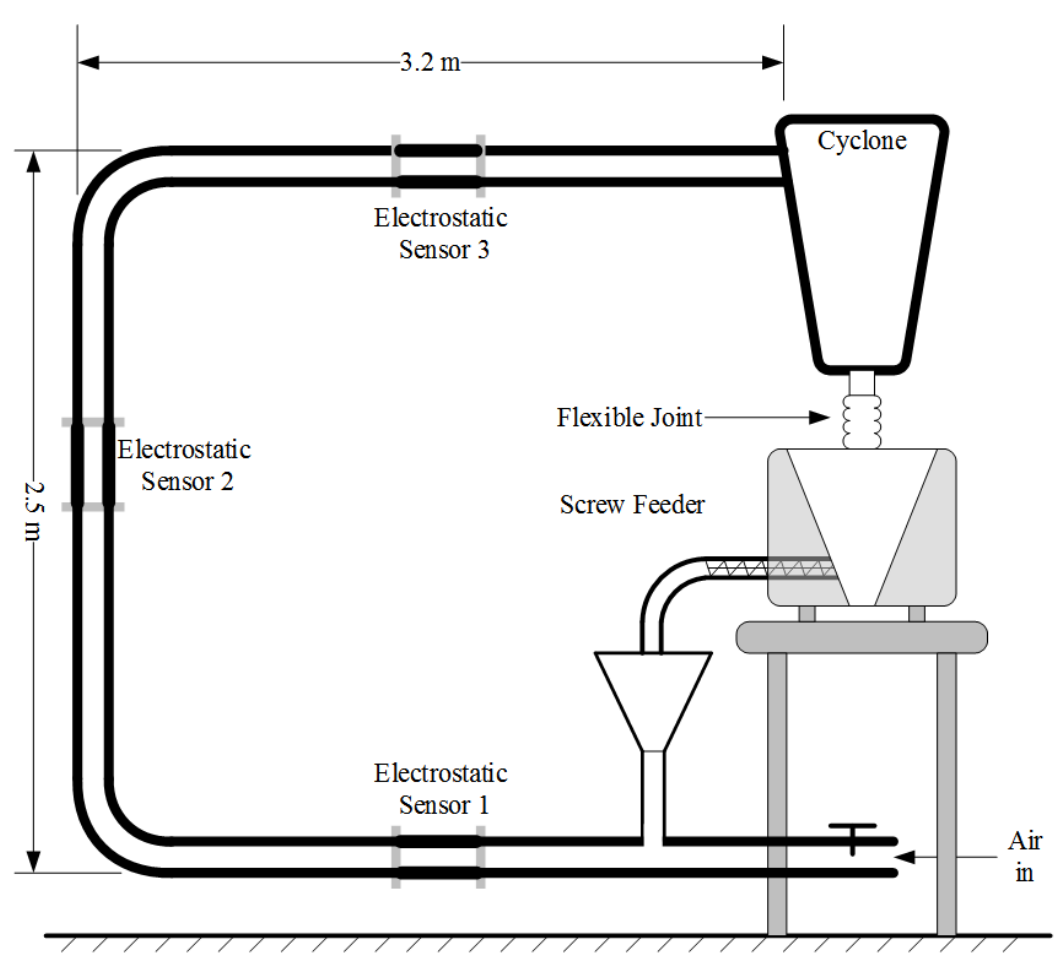

Fig. 2. Schematic of the pneumatic conveying experimental setup.

During conveying, three electrostatic sensors constantly monitored the flow. The resulting signals were subsequently acquired and transmitted to a computer. The mean solids flow velocity at each of the three measurement locations was produced based on the cross-correlation method (Beck \& Plaskowski, 1967; Coulthard, 1973; Keech, Coulthard, \& Cheng, 1998). The mean solids flow rate was indicated by the signal fluctuation level, as previously discussed. The airflow rate was also measured concurrently.

Fillite material was selected for the conveying test. Its material properties are shown in Table 1. Four different air velocities (12, 16, 22, and $26 \mathrm{~m} / \mathrm{s}$ ) at the inlet position were configured, by adjusting the inlet air mass flow rate. A screw feeder was used to feed $3 \mathrm{~kg}$ of the fillite material into the pipeline. Under the four air velocities, the screw feeder speed was regulated to maintain a constant air/solids mass flow rate ratio of 4 . Under each inlet air velocity, a number of conveying loops were completed before the sample was discharged, after which its particle size distribution was analysed.

Table 1. Experimental parameters.

\begin{tabular}{l|c}
\hline Material & Fillite \\
\hline Mean particle size, $d_{50}(\mu \mathrm{m})$ & 175 \\
\hline Particle density $\left(\mathrm{kg} / \mathrm{m}^{3}\right)$ & 900 \\
\hline Air/solids mass flow rate ratio & 4 \\
\hline Number of loops & $2,4,6,8$, and10 \\
\hline
\end{tabular}




\section{Influence of particle degradation on electrostatic sensor signals}

During the conveying tests, the mean particle sizes of the samples were analysed after 2, 4, 6, 8, and 10 loops of pneumatic transport. Fig. 3 shows the variation in particle size after different numbers of conveying loops, at the four inlet air velocities. The mean particle size decreased with increasing number of conveying loops, suggesting particle degradation due to vigorous particle-particle and particle-wall collisions and mechanical processes. No decrease in mean particle size was observed at an air inlet velocity of $12 \mathrm{~m} / \mathrm{s}$, suggesting negligible particle degradation under these conditions.

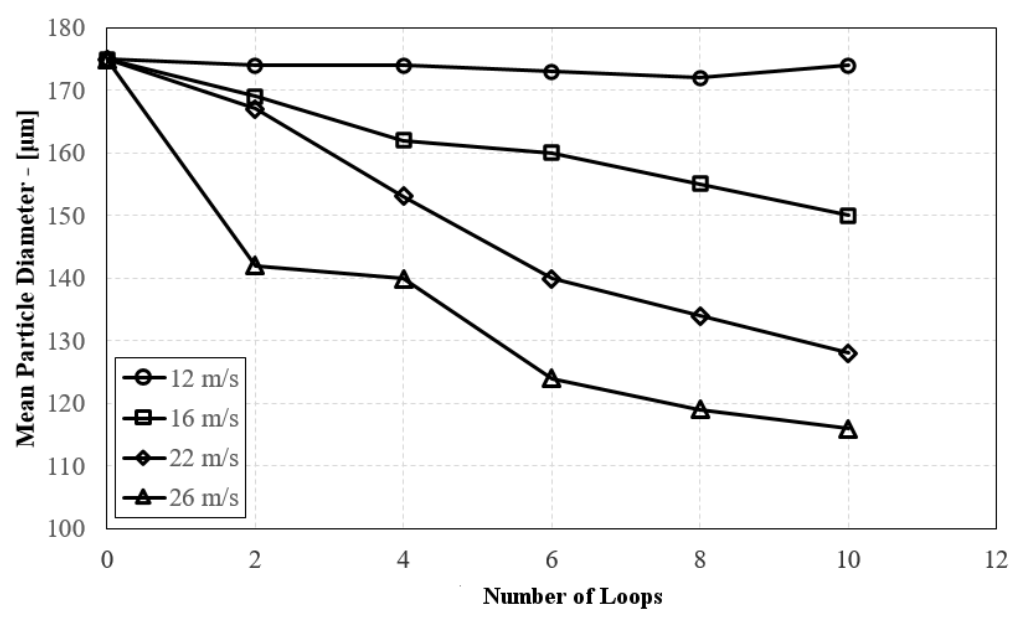

Fig. 3. Particle degradation with respect to conveying loop number, for four different air inlet velocities.

The electrostatic sensor responses were also collected under different conveying conditions. As previously discussed, the obtained signal was dynamic and exhibited fluctuation. The solids flow velocity was indicated by the root mean square value $\left(\widehat{U}_{\text {rms }}\right)$ of the voltage output signal. From the experiments, $\widehat{U}_{\text {rms }}$ varied with increasing number of conveying loops under a given air inlet velocity, as shown in Fig. 4. To define the change in $\widehat{U}_{\text {rms }}$, the following expression was used,

$$
\Delta \bar{U}=\frac{\widehat{U}_{\mathrm{rms}}(n)-\widehat{U}_{\mathrm{rms}}(1)}{\widehat{U}_{\mathrm{rms}}(1)} \times 100 \%,
$$

where $\widehat{U}_{\text {rms }}(n)$ is the root mean square value of the voltage output signal after completing $n$ conveying loops, and $\widehat{U}_{\text {rms }}(1)$ was is the root mean square value of the signal after the first conveying loop. Based on the aforementioned analysis, the mean particle size of the conveyed sample generally decreased with increasing number of conveying loops. Therefore, the variation in $\widehat{U}_{\text {rms }}$ can be plotted against the corresponding mean particle size after $n$ loops. From this, the influence of the particle size reduction on the electrostatic sensor signal could be revealed, as shown in Fig. 4. 


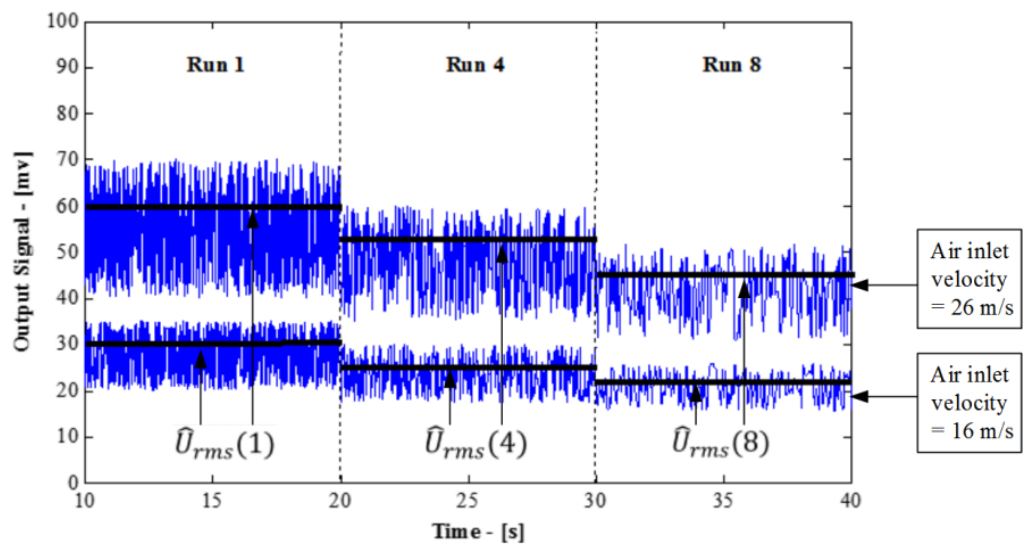

Fig. 4. Electrostatic sensor voltage outputs after conveying loop numbers 1, 4, and 8, for air inlet velocities of 16 and $26 \mathrm{~m} / \mathrm{s}$.

Fig. 5 shows the variation in $\widehat{U}_{\text {rms }}$ with the mean particle diameter after a specific number of conveying loops. At a low inlet air velocity of $12 \mathrm{~m} / \mathrm{s}$, the mean particle size and $\Delta \bar{U}$ exhibited minor fluctuations. At higher inlet air velocities of 16, 22, and $26 \mathrm{~m} / \mathrm{s}$, the mean particle size and signal level significantly decreased. The maximum signal level reductions at air inlet velocities of 16, 22 , and $26 \mathrm{~m} / \mathrm{s}$ were $13.5 \%$, 30.4\%, and 47.9\%, respectively.

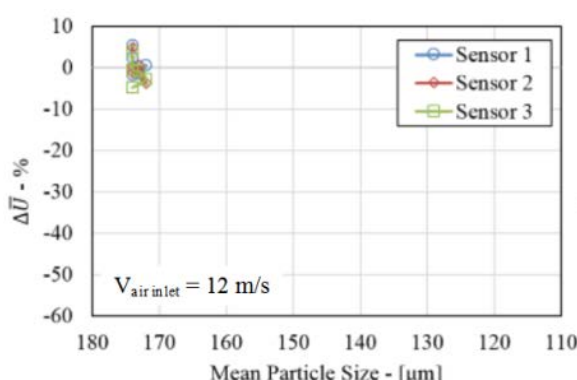

(a)

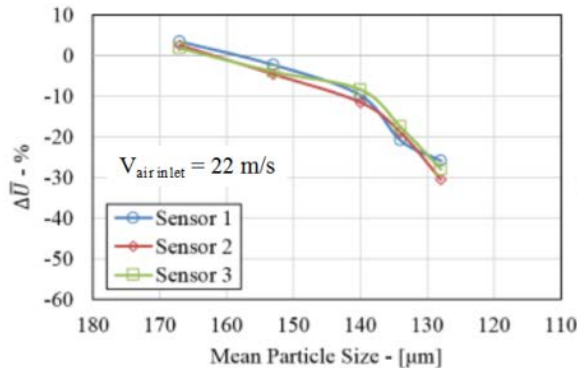

(c)

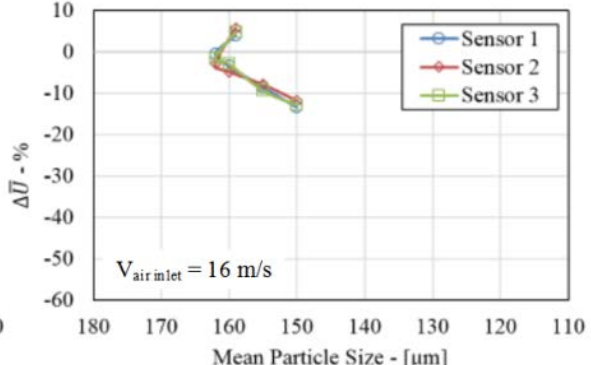

(b)

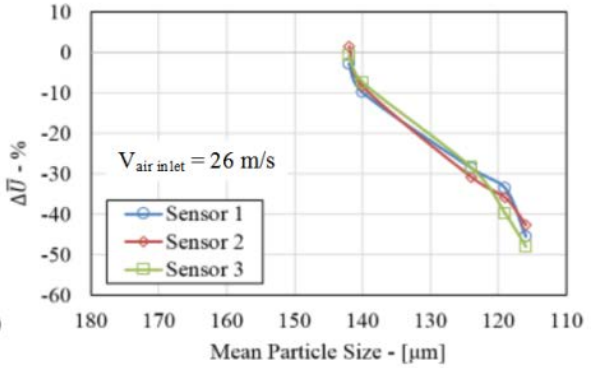

(d)

Fig. 5. Influence of particle size reduction on the variation in sensor output, for four different air inlet velocities.

The rise or fall of the signal level resulted from the combined effects of the total particle charge and flow velocity fluctuation during conveying. Therefore, it was concluded from Fig. 5 that no particle degradation occurred at a low air inlet velocity of $12 \mathrm{~m} / \mathrm{s}$, and thus the fluctuation in the electrostatic signal was minor. However, particle degradation was progressively more pronounced when increasing air inlet velocities of 16, 22, and $26 \mathrm{~m} / \mathrm{s}$ were applied. The associated reductions in electrostatic sensor signal levels were proportional to the degree of particle 
degradation. This suggested that the fluctuation in flow velocity fluctuation was lower with as the particle degradation increased.

\section{Numerical modelling methodology}

Coupled computational fluid dynamics and discrete element methods (CFD-DEM) analysis is a useful tool for studying dilute pneumatic conveying systems. In fluid dynamics, fluctuations in the flow velocity affect the readings of electrostatic sensors. The flow velocity fluctuations directly characterise the turbulence kinetic energy, which can be revealed using CFD-DEM modelling. The following experiments aimed to investigate the change in the turbulence kinetic energy due to particle degradation using the numerical method, to confirm the above-discussed experimental results.

To couple CFD with DEM, the fluid-particle (air-particle in this study) interactions must be properly modelled. Complex air-particle interaction forces, such as the buoyance force, drag force, pressure gradient, virtual mass force, lift force, etc. have been considered in numerous studies (Chu \& Yu, 2008; Goniva, Kloss, Hager, \& Pirker, 2010; Kloss, Goniva, Hager, Amberger, \& Pirker, 2012; Lim, Wang, \& Yu, 2006). These interaction forces need to be exchanged between CFD and DEM computations at the optimum time to achieve satisfactory modelling results. Specifically, particle motion is governed by Newton's law of motion, and is modelled by the DEM engine. The continuous air phase is described using the locally-averaged Navier-Strokes equations, which are solved by the CFD code.

For a particle modelled by the DEM, the following governing equations are used to define its motion,

$$
\left\{\begin{array}{c}
m \frac{d \mathrm{U}_{\mathrm{p}}}{d t}=\sum \mathrm{F}_{\mathrm{c}}+\mathrm{F}_{\mathrm{f}}+\mathrm{F}_{\mathrm{g}} \\
I \frac{d \omega}{d t}=\sum \mathrm{M}
\end{array},\right.
$$

where $\mathbf{U}_{\mathrm{p}}$ and $\boldsymbol{\omega}$ are the translational and angular velocities of the particle. $\mathbf{F}_{\mathrm{c}}$ and $\mathbf{M}$ are the contact force and torque, respectively, which are applied on particles from particle-particle and particle-wall contacts, and are computed by the Hertz-Mindlin contact model (Cundall, 1988). $\mathbf{F}_{\mathrm{f}}$ is the air-particle interphase momentum transfer force acting on the particle, $\mathbf{F}_{\mathrm{g}}$ is the gravitational force, and $m$ and $I$ are the mass and inertia of the particles, respectively.

In the CFD modelling, the meshing process discretised the continuous air phase domain into various cells. The air density, pressure, and velocity in each cell were locally averaged. For all cells in the entire mesh, the following continuity equation was applied,

$$
\frac{\partial\left(\varepsilon \rho_{\mathrm{a}}\right)}{\partial t}+\nabla \cdot\left(\varepsilon \rho_{\mathrm{a}} \mathbf{U}_{\mathrm{a}}\right)=0
$$


where $\mathbf{U}_{\mathrm{a}}$ is the average velocity of the fluid cell. $\varepsilon$ was is the void fraction of the cell, and is defined as the ratio of the void volume to the total volume of the cell. $\rho_{\mathrm{a}}$ is the air density. The formula defining the Navier-Strokes equation for each cell is,

$$
\frac{\partial\left(\varepsilon \rho_{\mathrm{a}} \mathbf{U}_{\mathrm{a}}\right)}{\partial t}+\nabla \cdot\left(\varepsilon \rho_{\mathrm{a}} \mathrm{U}_{\mathrm{a}} \mathbf{U}_{\mathrm{a}}\right)-\varepsilon \nabla \cdot\left(\mu_{\mathrm{a}} \nabla \mathbf{U}_{\mathrm{a}}\right)=-\nabla P-\mathbf{F}_{\mathrm{f}}+\varepsilon \rho_{\mathrm{a}} \mathbf{g}
$$

where $P$ was is the air pressure in the cell, $\mu_{\mathrm{a}}$ was is the cell air viscosity, $\mathbf{F}_{\mathrm{f}}$ is the air-particle interaction force, and g was is the gravitational acceleration.

In pneumatic conveying, the predominant air-particle interaction forces are the drag force $\mathbf{F}_{\mathrm{d}}$ and buoyance force $\mathbf{F}_{\mathrm{b}}$ (Sommerfeld \& Zivkovic, 1992). Numerous drag force models for air-particle interactions have been developed for CFD-DEM applications, including the Di Felice drag model (Di Felice, 1994), Wen Yu drag model (Wen \& Yu, 1966), and Hill-Koch-Ladd drag model (Benyahia, Syamlal, \& O’Brien, 2006; Hill, Koch, \& Ladd, 2001). The Ergun Wen Yu drag model (Gidaspow, 2012) was employed in the current study, because of its suitability to a wide range of particle packing conditions. The expression for the drag force is,

$$
\mathbf{F}_{\mathrm{d}}=\left\{\begin{array}{ll}
\frac{3}{4} \frac{C_{\mathrm{D}} \varepsilon(1-\varepsilon) \rho_{\mathrm{a}}\left|\mathbf{U}_{\mathrm{a}}-\mathbf{U}_{\mathrm{p}}\right|}{d_{\mathrm{p}}} \varepsilon^{-2.65} & \varepsilon>0.8 \\
150 \frac{\mu_{\mathrm{a}}(1-\varepsilon)^{2}}{\varepsilon^{2} d_{\mathrm{p}}{ }^{2}}+1.75 \frac{\rho_{\mathrm{a}}(1-\varepsilon)}{\varepsilon d_{\mathrm{p}}}\left|\mathbf{U}_{\mathrm{a}}-\mathbf{U}_{\mathrm{p}}\right| & \varepsilon \leq 0.8
\end{array},\right.
$$

where $C_{\mathrm{D}}$ is the drag coefficient and is defined as,

$$
C_{\mathrm{D}}=\left\{\begin{array}{ll}
\frac{24}{\varepsilon R e}\left[1+0.15(\varepsilon R e)^{0.687}\right] & \varepsilon R e<1000 \\
0.44 & \varepsilon R e \geq 1000
\end{array},\right.
$$

where $R e$ is the Reynolds number. The buoyance force is defined as,

$$
\mathbf{F}_{\mathrm{b}}=\frac{1}{6} \pi \rho_{\mathrm{a}} d_{\mathrm{p}}^{3} \mathbf{g}
$$

The CFD-DEM computational process is shown in Fig. 6. The unresolved CFD-DEM method was employed, which used a CFD mesh size of a few times larger than the particle size. At the beginning of the computation, the DEM calculated the position and velocity of each particle in the system. The updated position of each particle was then characterised into relevant cells in the CFD mesh, from which the Navier-Stroke equations for all cells were then solved by the CFD toolbox. The drag and buoyance forces to be applied on the particles were also determined by the resulting air pressure and velocity in a specific cell. Providing the CFD computation converged, the drag and buoyance forces were then transferred to the DEM engine for re-iteration. 


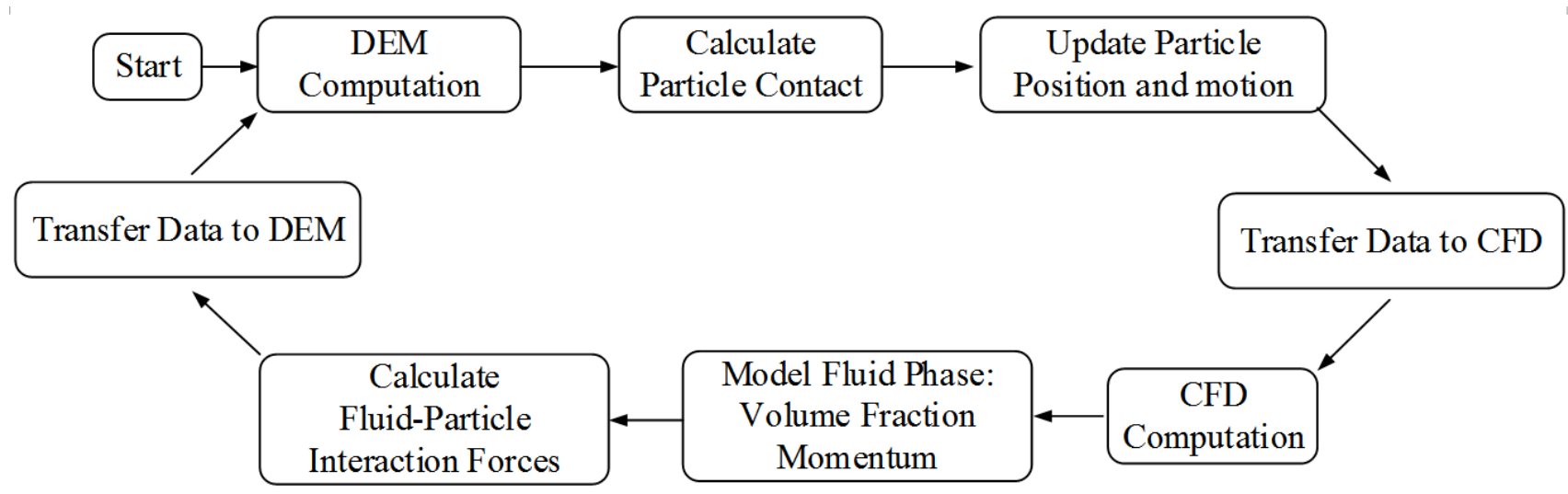

Fig. 6. Flowchart of the CFD-DEM computation process.

The open source code LIGGGHTS (Kloss \& Goniva, 2010) was selected as the DEM modelling package, and OpenFOAM (Jasak, Jemcov, \& Tukovic, 2013) was used as the CFD solver. To couple the OpenFOAM solver with the LIGGGHTS code, a customised library was developed and incorporated into the numerical modelling packages. The rhoPimpleFoam solver was used in the OpenFOAM to solve the locally averaged Navier-Strokes equations.

\subsection{Numerical modelling setup}

Direct modelling of the particle degradation is a complex process and is beyond the scope of the current study. Nonetheless, to reflect the particle degradation occurring in the experiments, an alternative method was used to conduct various simulations using different mono-sized particles. The influence of particle degradation on the turbulence kinetic energy (flow velocity fluctuation) in each simulation could then be qualitatively assessed. The principle of this method is illustrated in Fig. 7.

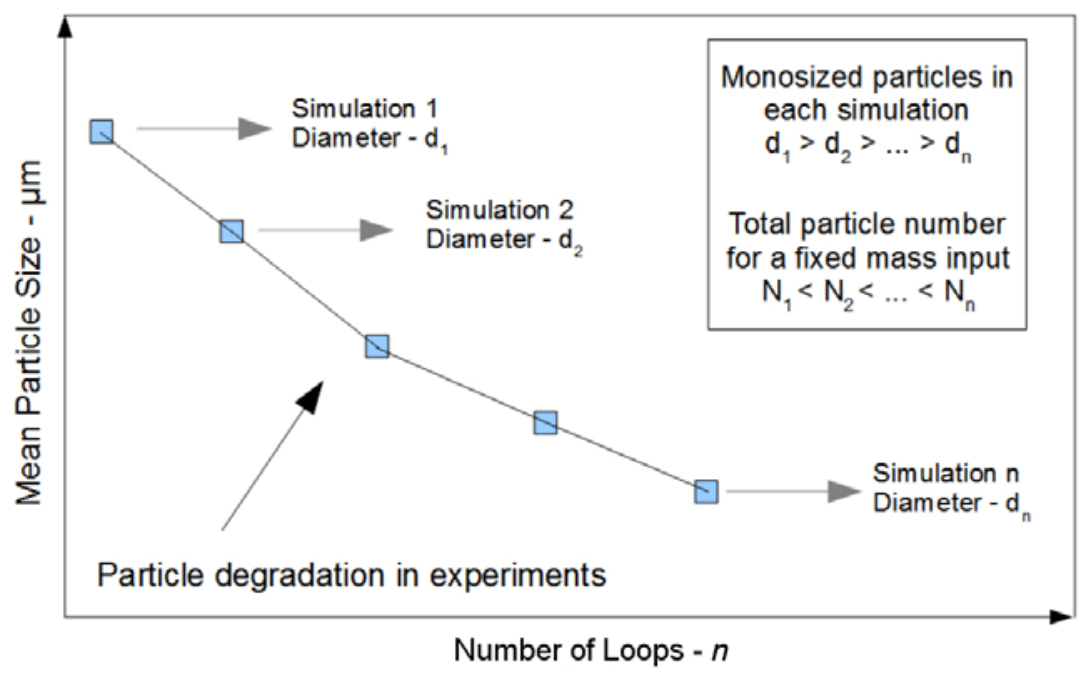

Fig. 7. Numerical modelling setup used to reflect the particle degradation. 
The maximum range of the degraded particle diameter in the experiments was set from 110 to $175 \mu \mathrm{m}$ under the air inlet velocity of $26 \mathrm{~m} / \mathrm{s}$, and was used for comparison with numerical modelling results. This size range was too small to achieve a realistic computational turnaround time. Therefore, scaled up particle diameters of 1.7, 1.5, 1.4, 1.3, and $1.2 \mathrm{~mm}$ were used in five simulations. This range was approximately 10 times wider than the actual mean particle diameters obtained in the experiments after the repeating conveying tests. A pipeline mesh was generated by scaling up 10 times of the experimental geometry, to maintain a constant pipe diameter to particle size ratio.

All numerical modelling parameters for both the DEM and CFD components are shown in Table 2. The general material property parameters in the DEM phase were kept the same throughout all five simulations, to eliminate their influence on the flow characteristics. These parameters were not calibrated to the actual material in the experiment, but were sufficient for a qualitative assessment. The electrostatic charge on the particles was neglected in the simulations, because of the minimum influence of the electrostatic force on the particle-particle and particle-fluid interactions (Yao, Zhang, Wang, Matsusaka, \& Masuda, 2004).

Table 2. DEM and CFD numerical modelling parameters.

\begin{tabular}{|c|c|c|}
\hline \multirow{14}{*}{$\begin{array}{l}\text { DEM } \\
\text { settings }\end{array}$} & Particle density $\left(\mathrm{kg} / \mathrm{m}^{3}\right)$ & 900 \\
\hline & Interparticle friction coefficient & 0.5 \\
\hline & $\begin{array}{l}\text { Particle rolling friction (Iwashita \& Oda, 1998; Wensrich } \\
\text { et al., 2014; Wensrich \& Katterfeld, 2012) }\end{array}$ & 0.1 \\
\hline & Wall friction coefficient & 0.3 \\
\hline & Restitution coefficient & 0.3 \\
\hline & Poisson’s ratio & 0.3 \\
\hline & Young’s modulus (GPa) & 70 \\
\hline & Material mass (kg) & 1.0 \\
\hline & Total particle number in respect to particle diameter & \\
\hline & $1.7 \mathrm{~mm}$ case & 431,929 \\
\hline & $1.5 \mathrm{~mm}$ case & 628,760 \\
\hline & $1.4 \mathrm{~mm}$ case & 773,348 \\
\hline & $1.3 \mathrm{~mm}$ case & 865,893 \\
\hline & $1.2 \mathrm{~mm}$ case & $1,228,047$ \\
\hline \multirow{6}{*}{$\begin{array}{l}\text { CFD } \\
\text { settings }\end{array}$} & Air inlet velocity (m/s) & 26 \\
\hline & Operating temperature $(\mathrm{K})$ & 300 \\
\hline & Gravitational acceleration $\left(\mathrm{m} / \mathrm{s}^{2}\right)$ & 9.81 \\
\hline & Inlet boundary condition & Velocity \\
\hline & Outlet boundary condition & Outflow \\
\hline & Turbulence model & $\begin{array}{l}\text { Standard } k K k- \\
\varepsilon\end{array}$ \\
\hline
\end{tabular}




\begin{tabular}{lll}
\hline Simulation & Time step (DEM) (s) & $5 \times 10^{-7}$ \\
control & Time step (CFD) (s) & $5 \times 10^{-4}$ \\
\hline
\end{tabular}

\section{Numerical modelling results}

Five simulations were performed following the numerical method discussed above, and the results are shown in Fig. 8. The flow velocity fluctuation was represented by the turbulence kinetic energy. The turbulence kinetic energies in each simulation when the particles passed sensors one, two, and three are shown in Fig. 8(a), (b), and (c), respectively. The corresponding particle flow velocities and dispersion patterns are also shown. The turbulence kinetic energy exhibited an attenuating trend when the particle size was reduced in the simulation. This qualitative observation was consistent throughout the results for the three sensors. This trend was consistent with the experimental results. This suggested that the decreasing electrostatic sensor outputs were due to the lower flow velocity fluctuation induced by particle degradation during dilute pneumatic conveying.

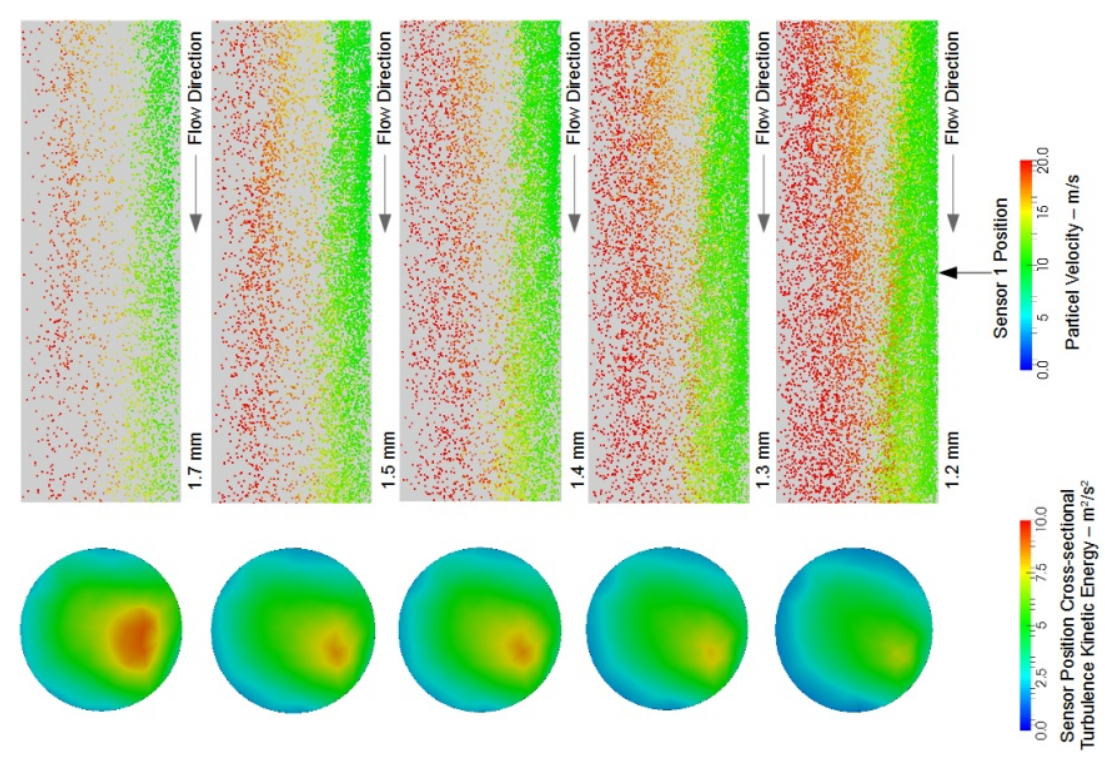

(a) 


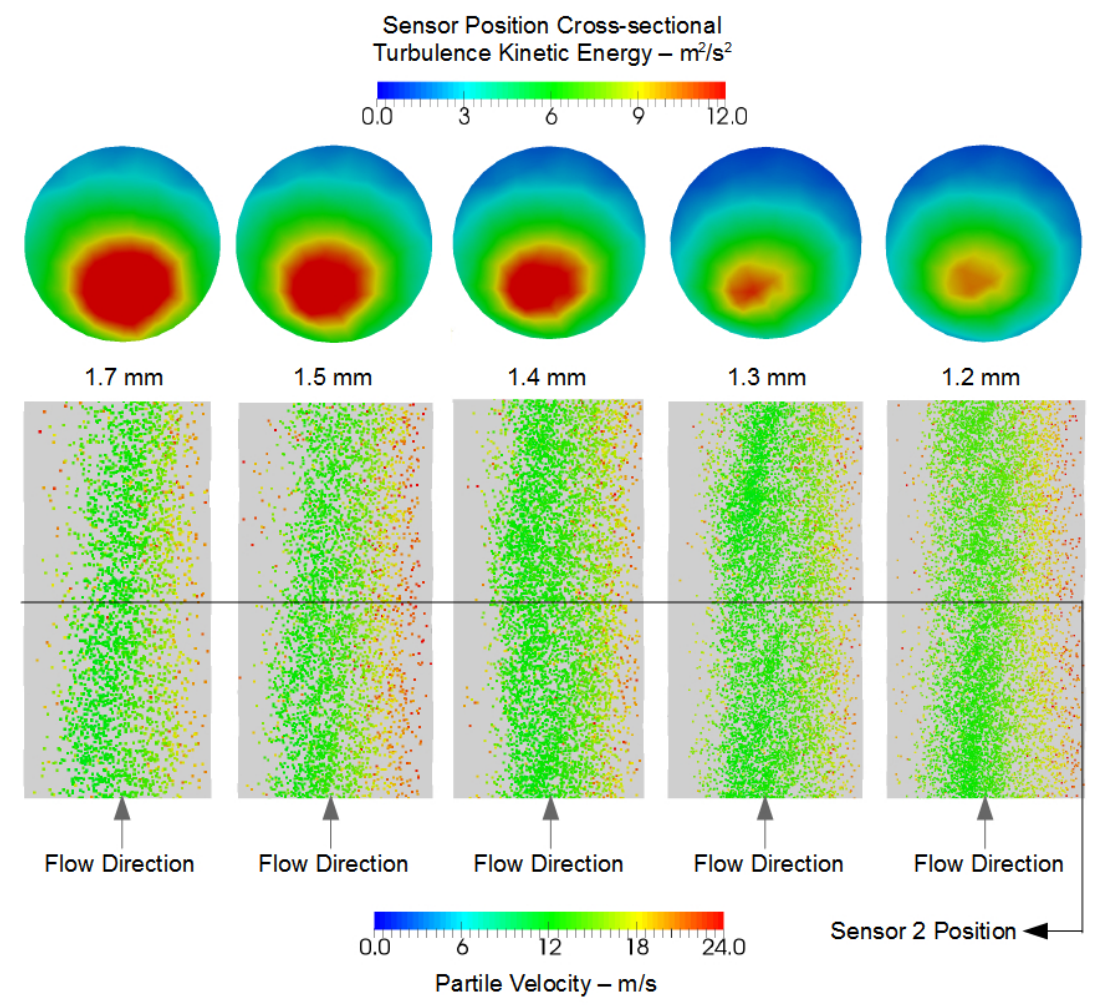

(b)

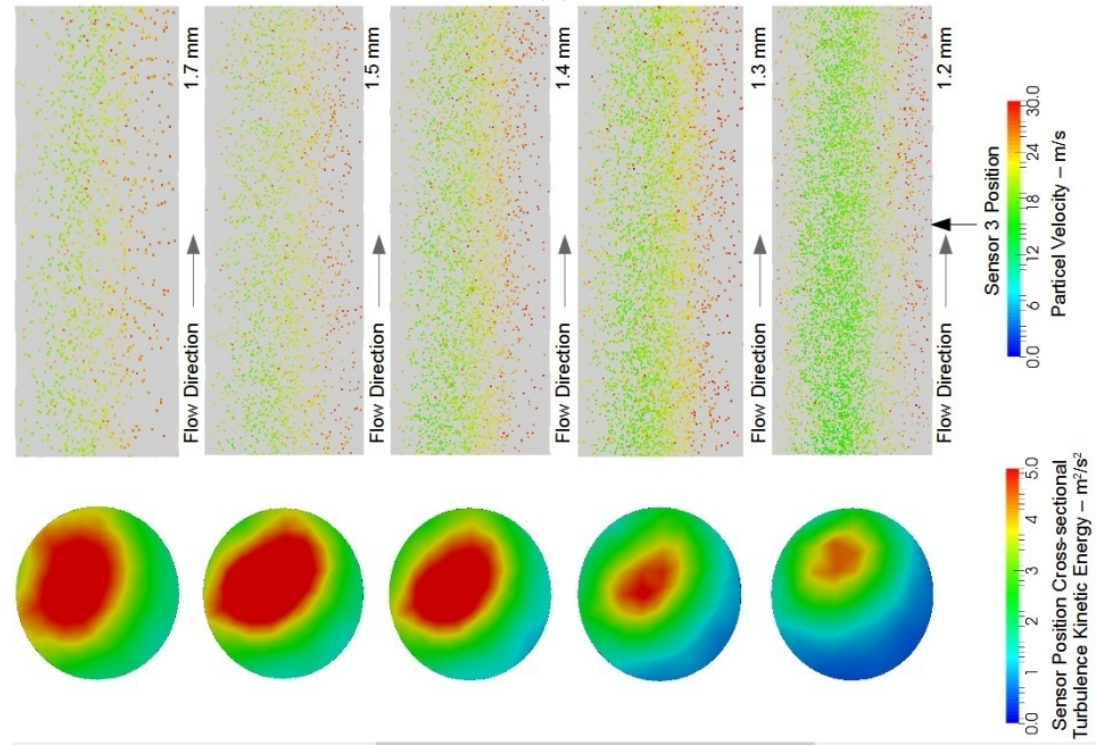

(c)

Fig.8. Turbulence kinetic energies and particle flow velocities when passing (a) sensor one, (b) sensor two, and (c) sensor three, in each simulation using mono-sized particles of sizes as stated.

The effect of particle size on gas-solid flow characteristics has been studied extensively (Jones, 2001; Lee \& Durst, 1982; Maeda, Hishida, \& Furutani, 1980; Tsuji, Morikawa, \& Shiomi, 1984). Contrasting results on the effect of particle size on flow turbulence modulation have been reported (Hadinoto \& Curtis, 2009). The turbulence modulation is largely related to the turbulence kinetic energy. A reported qualitative numerical modelling study suggested the attenuation of the turbulence kinetic energy with decreasing particle size (Crowe, 2000; Hetsroni, 1989). 


\section{Conclusions}

This study investigated the effect of particle degradation on electrostatic sensor measurements and flow characteristics in dilute pneumatic conveying pipelines. The experimental and numerical results yield the following findings:

- Rapid air-fillite flow in dilute pneumatic conveying pipelines led to particle degradation. The degree of particle degradation was proportional to the air inlet velocity.

- Particle degradation induced a decrease in the signal level during the electrostatic sensor measurements. This resulted in the electrostatic sensor measurement being directly affected by the flow velocity fluctuation. Particle degradation was thought to result in the variation of flow velocity fluctuation in the air-fillite flow.

- Qualitative numerical modelling of the air-solids flow suggested that the turbulence kinetic energy attenuated with decreasing particle size in the two-phase flow. The turbulence kinetic energy was characterized by the flow velocity fluctuation. The numerical modelling validated the experimental observations that particle degradation led to a decrease in the signal level of electrostatic sensors in the dilute air-fillite pneumatic flow.

These findings provide important information for accurate flow measurement using electrostatic flow meters. During practical application, the product quality was deemed to be maintained, in order for the electrostatic sensor to produce consistent readings. The variation in product quality would require re-calibration of the electrostatic flow meters. A future in-depth investigation on the effect of particle size on the variation in electrostatic charge in rapid dilute pneumatic flow should be conducted.

\section{References}

Arko, A., Waterfall, R. C., Beck, M. S., Dyakowski, T., Sutcliffe, P., \& Byars, M. (1999). Development of electrical capacitance tomography for solids mass flow measurement and control of pneumatic conveying systems. In 1st World Congress on Industrial Process Tomography (pp. 14-17).

Beck, M. S., Green, R. G., \& Thorn, R. (1987). Non-intrusive measurement of solids mass flow in pneumatic conveying. Journal of Physics E: Scientific Instruments, 20(7), 835-840.

Beck, M. S., \& Plaskowski, A. (1967). How Inherent flow noise can be used to measure mass flow of granular materials. Instrument Review, 458-461.

Benyahia, S., Syamlal, M., \& O’Brien, T. J. (2006). Extension of Hill-Koch-Ladd drag correlation over all ranges of Reynolds number and solids volume fraction. Powder Technology, 162(2), 166-174.

Chapelle, P., Abou-Chakra, H., Christakis, N., Patel, M., Abu-Nahar, A., \& Tüzün, U., et al. (2004). Computational model for prediction of particle degradation during dilute-phase pneumatic conveying: the use of a laboratoryscale degradation tester for the determination of degradation propensity. Advanced Powder Technology, 15(1), 
$13-29$.

Chu, K. W., \& Yu, A. B. (2008). Numerical simulation of the gas-solid flow in three-dimensional pneumatic conveying bends. Industrial \& Engineering Chemistry Research, 47(18), 7058-7071.

Cole, B. N., Baum, M. R., \& Mobbs, F. R. (1969). An investigation of electrostatic charging effects in high-speed gas-solids pipe flows. In Proceedings of the Institution of Mechanical Engineers, Conference Proceedings (Vol. 184, pp. 77-83). SAGE Publications.

Coulthard, J. (1973). Ultrasonic cross-correlation flowmeters. Ultrasonics, 11(2), 83-88.

Crowe, C. T. (2000). On models for turbulence modulation in fluid-particle flows. International Journal of Multiphase Flow, 26(5), 719-727.

Cundall, P. A. (1988). Computer simulations of dense sphere assemblies. Micromechanics of Granular Materials, 4, $113-123$.

Di Felice, R. (1994). The voidage function for fluid-particle interaction systems. International Journal of Multiphase Flow, 20(1), 153-159.

Farmer, A. D. (1992). Triboelectricity—A tool for solids flow measurement. Ceramics. Adding the Value., 1, 113117.

Gajewski, J. B. (1997). Electric charge measurement in pneumatic installations. Journal of Electrostatics, 40, 231236.

Gajewski, J. B., \& Szaynok, A. (1981). Charge measurement of dust particles in motion. Journal of Electrostatics, 10, 229-234.

Gidaspow, D. (2012). Multiphase flow and fluidization: Continuum and kinetic theory descriptions. New York: Academic press.

Goniva, C., Kloss, C., Hager, A., \& Pirker, S. (2010). An open source CFD-DEM perspective. In Proceedings of OpenFOAM workshop (pp. 22-24), Gothenburg, Sweden.

Hadinoto, K., \& Curtis, J. S. (2009). Reynolds number dependence of gas-phase turbulence in particle-laden flows: Effects of particle inertia and particle loading. Powder Technology, 195(2), 119-127.

Hetsroni, G. (1989). Particles-turbulence interaction. International Journal of Multiphase Flow, 15(5), 735-746.

Hill, R. J., Koch, D. L., \& Ladd, A. J. C. (2001). Moderate-Reynolds-number flows in ordered and random arrays of spheres. Journal of Fluid Mechanics, 448, 243-278. 
Iwashita, K., \& Oda, M. (1998). Rolling resistance at contacts in simulation of shear band development by DEM. Journal of Engineering Mechanics, 124(3), 285-292.

Jasak, H., Jemcov, A., \& Tukovic, Z. (2013). OpenFOAM: A C++ library for complex physics simulations. In International Workshop on Coupled Methods in Numerical Dynamics (Vol. 1000, pp. 1-20). IUC Dubrovnik, Croatia.

Jones, E. N. (2001). An experimental investigation of particle size distribution effect in dilute-phase gas-solid flow. Doctoral dissertation, Purdue University, USA.

Kalman, H. (2000). Attrition of powders and granules at various bends during pneumatic conveying. Powder Technology, 112(3), 244-250.

Keech, R., Coulthard, J., \& Cheng, R. (1998). Measurement using cross correlation. WO/1998/055839.

King, P. W. (1973). Mass flow measurement of conveyed solids by monitoring of intrinsic electrostatic noise levels. In Proceedings of the 2nd International Conference on the Pneumatic Transport of Solids in Pipes, Cranfield.

Kloss, C., \& Goniva, C. (2010). LIGGGHTS: A new open source discrete element simulation software. In Proceedings of the 5th International Conference on Discrete Element Methods (pp. 25-26).

Kloss, C., Goniva, C., Hager, A., Amberger, S., \& Pirker, S. (2012). Models, algorithms and validation for opensource DEM and CFD-DEM. Progress in Computational Fluid Dynamics, an International Journal, 12(2), 140-152.

Lee, S. L., \& Durst, F. (1982). On the motion of particles in turbulent duct flows. International Journal of Multiphase Flow, 8(2), 125-146.

Lim, E.W.C., Wang, C.H., \& Yu, A.B. (2006). Discrete element simulation for pneumatic conveying of granular material. AIChE Journal, 52(2), 496-509.

Maeda, M., Hishida, K., \& Furutani, T. (1980). Velocity distributions of air-solids suspension in upward pipe flow. (Effect of particles on air velocity distribution). Translation of the Japan Society of Mechanical Engineerings, Series B, 46, 2313-2320. (in Japanese)

Masuda, H., Komatsu, T., Mitsui, N., \& Iinoya, K. (1977). Electrification of gas-solid suspensions flowing in steel and insulating-coated pipes. Journal of Electrostatics, 2(4), 341-350.

Mills, D., Jones, M. G., \& Agarwal, V. K. (2004). Handbook of pneumatic conveying engineering. Boca Raton: CRC Press.

Saleh, K., \& Aghili, A. (2012). The spatio-temporal evolution of tribo-electric charge transfer during the pneumatic 
conveying of powders: Modelling and experimental validation. Chemical Engineering Science, 68(1), 120-131.

Salman, A. D., Attila, V., \& Mills, D. (1992). Particle degradation in dilute phase pneumatic conveying systems. In Proceedings of the Technical Program International Powder and Bulk Solids Handling and Processing (Vol. 17, pp. 337).

Sommerfeld, M., \& Zivkovic, G. (1992). Recent advances in the numerical simulation of pneumatic conveying through pipe systems. In C. Hirsch (ed.), Computational methods in applied sciences (pp. 201-212).

Tsuji, Y., Morikawa, Y., \& Shiomi, H. (1984). LDV measurements of an air-solid two-phase flow in a vertical pipe. Journal of Fluid Mechanics, 139, 417-434.

Wen, C.Y., \& Yu, Y. H. (1966). Mechanics of fluidization. Chemical Engineering Process Symposium Series, 62(2), 100-111.

Wensrich, C. M., \& Katterfeld, A. (2012). Rolling friction as a technique for modelling particle shape in DEM. Powder Technology, 217, 409-417.

Wensrich, C. M., Katterfeld, A., \& Sugo, D. (2014). Characterisation of the effects of particle shape using a normalised contact eccentricity. Granular Matter, 16(3), 327-337.

Xu, C., Zhou, B., \& Wang, S. (2010). Dense-phase pneumatically conveyed coal particle velocity measurement using electrostatic probes. Journal of Electrostatics, 68(1), 64-72.

Yan, Y. (1996). Mass flow measurement of bulk solids in pneumatic pipelines. Measurement Science and Technology, 7(12), 1687-1706.

Yan, Y., Byrne, B., \& Coulthard, J. (1995). Sensing field homogeneity in mass flow rate measurement of pneumatically conveyed solids. Flow Measurement and Instrumentation, 6(2), 115-119.

Yao, J., Zhang, Y., Wang, C.-H., Matsusaka, S., \& Masuda, H. (2004). Electrostatics of the granular flow in a pneumatic conveying system. Industrial \& Engineering Chemistry Research, 43(22), 7181-7199.

Yarin, L. P., \& Hetsroni, G. (1994). Turbulence intensity in dilute two-phase flows-1 Effect of particle-size distribution on the turbulence of the carrier fluid. International Journal of Multiphase Flow, 20(1), 1-15.

Zhang, J., \& Coulthard, J. (2005). Theoretical and experimental studies of the spatial sensitivity of an electrostatic pulverised fuel meter. Journal of Electrostatics, 63(12), 1133-1149.

Zhang, J., Coulthard, J., Cheng, R., \& Keech, R. (2009). Measuring pulverised fuel: Using electrostatic meters. Measurement and Control, 42(3), 87-90. 
Zhou, B., Zhang, J., Xu, C., \& Wang, S. (2011). Image reconstruction in electrostatic tomography using a priori knowledge from ECT. Nuclear Engineering and Design, 241(6), 1952-1958. 